\title{
Epidemiology of rotavirus gastroenteritis
}

\author{
Y D SENTURIA \\ From the Department of Paediatric Epidemiology, Institute of Child Health, 30 Guilford Street, London WC1N \\ $1 E H$
}

Infantile gastroenteritis and the resulting dehydrating diarrhoea is a major cause of morbidity in developed countries and of mortality in many developing countries. Since their original description in 1973, rotaviruses have emerged as the single most important cause of diarrhoea in infants and young children requiring admission to hospital for treatment of gastroenteritis. ${ }^{1}$ A desire to reduce the significant morbidity associated with rotaviruses led to the development of a live attenuated vaccine, ${ }^{2}$ recently made available for trials in this country. Concurrently, advances in adequate typing systems allowing characterisation of virus from different geographic areas and from sequential infections in populations followed longitudinally have increased our understanding of the epidemiology of this most important group of viruses. ${ }^{3}$ Knowledge of the epidemiology of rotavirus would be important in the planning of any vaccine programme contemplated for this country.

The gastroenteritis associated with rotavirus has consistently been described as more severe than that caused by other agents. The pathophysiology is felt to be loss of absorptive capacity in the small intestine. The incubation period is 48 hours, with viral excretion preceding the onset of symptoms. There is often early vomiting followed by explosive and watery diarrhoea lasting 5-7 days. Virus is usually shed from the 3 rd-8th days. Although IgM antibody appears on the 5th day and stays elevated for about three weeks, IgG antibody does not appear until 2-4 weeks after infection. ${ }^{4}$

Cross-sectional seroepidemiological surveys have shown high antibody levels in the newborn (due to transfer of passive antibody from mother), falling over the first six months of life, peaking again from 2-3 years. High geometric mean titres are then maintained until the age of 40 when levels begin a gradual decline. ${ }^{5}$

Children aged 6-24 months seem most susceptible to clinical illness following rotavirus infection with peak incidence in most series at 9-12 months. Sequential rotavirus illness can occur in the same child, although such illnesses are characteristically due to different serotypes. A large prospective study in Washington reported 3.7 episodes of rotavirus gastroenteritis per 1000 infants per epidemic year with 2.2 episodes $/ 1000 /$ year in the $12-24$ month age group. Rotavirus was implicated in $50 \%$ of hospitalised episodes of diarrhoea in children under the age of two. ${ }^{6}$ A prospective study from Copenhagen similarly found rotavirus to be the most frequent pathogen in children hospitalised with gastroenteritis. Incidence was highest under the age of 12 months, with twice as many cases between $6-12$ as between 0-6 months. ${ }^{7}$ Both series noted a decline in incidence of hospitalisation and outpatient visits with age, and a predominance of males among hospitalised patients.

Transmission of rotaviruses is assumed to be fecaloral and probably varies with climate, population density and local habits. In temperate climates, rotavirus gastroenteritis peaks in the cooler months, as illustrated by the series from Copenhagen where $85 \%$ of isolations occurred from January-April. A similar seasonal trend was noted in Tecumseh. ${ }^{8}$ In contrast, this virus is seen year round in most tropical climates.

In prospective follow-up of children from 20 daycare centres in Houston, rotavirus was recovered exclusively from children under the age of 3 , with a secondary attack rate of $15 \% .^{9}$ A subsequent prospective study in Arizona day-care centres found rotavirus significantly more often in children under 12 months, compared to those 13-36 months of age $(p<0.001){ }^{10}$ Intra-familial transmission was emphasised by a series from New Zealand: 28 families exposed to a child with rotavirus were studied prospectively. $75 \%$ of siblings under the age of 12 acquired rotavirus, in contrast to control families (exposed to non-rotavirus gastroenteritis) where there were no secondary cases of diarrhoea. Even $33 \%$ of adults exposed to rotavirus through index children experienced serological conversions, although their infections were more likely to be mild or subclinical. ${ }^{11}$

The rotavirus genome consists of 11 segments of double stranded RNA. Rotavirus is identified initially by electron microscopy and blocking ELISA on stool samples. Three major forms of serological classification of strains have emerged. Reactions of sera against major inner capsular antigen distinguish at least two subgroups. Neutralisation by 
hyperimmune sera distinguishes $2-5$ serotypes. The large variety of genomic patterns exhibited by human rotaviruses has permitted the identification of long and short electropherotypes by migration patterns of nucleic acid in gel on silver staining. ${ }^{3}$ Strains showing long pattern on electrophoresis tend to correspond to subgroup 1 and serotype 2 .

Prevalence of subgroups in an area tends to vary from year to year. In Washington, the rate of isolation of serotype 2 decreased from $100 \%$ in 1973-1977 to $57 \%$ in $1977-1978$ while in the West Midlands only $10 \%$ of isolates were serotype 2 from 1978-1982, increasing to $70 \%$ in $1982-1983 .{ }^{12}$ Even different regions of the same country may show differing prevalences during the same epidemic season. While short electropherotypes were virtually absent in Glasgow in 1982-1983, they predominated in London and the West Midlands during the same epidemic year. ${ }^{13}$

A three year survey in Brussels found simultaneous occurrence of various subgroups of virus in constant proportions even during the annual winter peak of gastroenteritis. ${ }^{14}$ In contrast, Japanese investigators reported a single dominant electropherotype during the first two months of an epidemic with various types found later on. ${ }^{15}$ Their hypothesis of antigenic drift was supported by the emergence of variants of the predominant electropherotypes during the Glasgow 1981-82 epidemic. ${ }^{16}$ This phenomenon is reminiscent of the behaviour of influenza strains, which also possess a segmented RNA genome.

Recovery of rotavirus from faeces can not be taken alone as diagnostically significant. A prospective series from a Paris hospital showed that $48 \%$ of their stool isolations represented asymptomatic rotavirus shedding with positive correlation between diarrhoea and virus shedding only in those cases of diarrhoea associated with fever and vomiting. ${ }^{17}$ Increasing age led to a decreasing proportion of carriers (positive stool EM and blocking ELISA, negative serology) and an increasing proportion of true infections (positive stool and sera with diarrhoea). This raised the aetiological issue of evolving intestinal maturity, further supported by finding six infants with initially low IgM and high IgG who had a late increase in IgM accompanied by diarrhoea as their IgG titre was declining. ${ }^{18}$ This was most compatible with infection acquired in hospital presumably as maternal immunity was waning.

Animal studies have consistently indicated that antibody in the lumen of the small intestine was of major importance in resistance to rotavirus disease. This pivotal role for local intestinal IgA antibody has been reinforced by studies in adult human volunteers. It was therefore postulated that maternal secretory IgA transmitted through breast milk might be protective. However, a prospective study of rotavirus gastroenteritis in Rochester showed no difference in age of infection, duration of diarrhoea or severity of diarrhoea between breast-fed and bottle-fed infants. ${ }^{19}$

Public health and hospital microbiology laboratories in England and Wales send reports of rotavirus isolations to the PHLS Communicable Disease Surveillance Centre. Four-weekly summaries appear in the Communicable Disease Report (PHLS CDSC unpublished). Quarterly statistics also appear in the OPCS Monitor on Infectious Diseases. ${ }^{20}$ Although the volume of reports has increased (from 4413 in 1981-1982 to 6401 in 1984-1985) there have been only minor variations in the distribution by age, region or season.

Review of CDSC data confirms that rotavirus is primarily a disease of infancy and early childhood, with $42 \%$ of notifications in the first year and $83 \%$ under the age of 5 (PHLS CDSC unpublished). Month-by-month analyses are not available to confirm the 9-12 month peak in the age distribution observed in other industrialised countries.

Each year the number of rotavirus reports to CDSC begins to rise during weeks $37-40$ and reaches its peak in the first quarter of the new year (figure). Examination of the CDSC reports by region shows that similar seasonal time trends occur in all areas of the country. In the last three epidemic years the initial excess isolations have come from Yorkshire and the North Western region, usually in weeks $41-44$ (PHLSCDSC unpublished). Comparing observed cases in each region with those expected based on population, there was a statistically significant $(p<0.05)$ excess of isolations for Yorkshire, North West Thames, the West Midlands and North Western regions during the 1983-1984 epidemic year. This could not be explained by differences in age distribution as the proportion under age 5 shows little variation between the regions (6-6.7\%). Adjusting the expected figures for reporting bias (using the proportion of total viral notifications coming from each region) still showed higher than

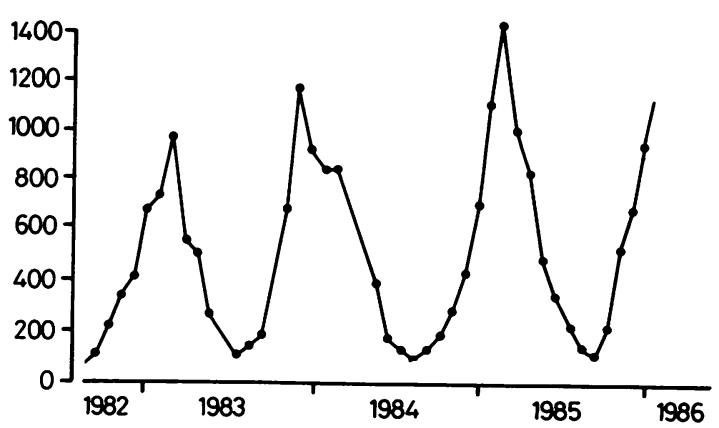

Four-weekly summaries of rotavirus notification, England and Wales, 1982-5 (PHLS-CDSC, unpublished data). 
expected isolations from Yorkshire, North West Thames, and the West Midlands. The overall increase in isolations in the past two years has not led to significant changes in ranking of $\mathrm{O} / \mathrm{E}$ ratios.

A live attenuated rotavirus vaccine containing subgroup 1 virus was initially tested in 20 seronegative Finnish adults, with no virus excretion or clinical symptoms. It was further tested on seronegative and seropositive Finnish 2 year olds with similarly favourable results and seroconversion rate of $70 \% .{ }^{21}$ A subsequent randomised double-blind placebocontrolled trial in 178 infants aged 8-11 months during an epidemic of subgroup 2 virus in Tampere showed $88 \%$ efficacy against natural challenge by wild rotavirus. $^{22}$ The same investigators then showed that seroconversion could be increased to $88 \%$ by simple milk feeding vaccination. ${ }^{23}$

Although the results from Finland are promising and show that the vaccine is safe and appears to be effective,${ }^{24}$ rotavirus vaccine trials in this country must address the efficacy in our population. The aim would be a small-scale field trial to evaluate efficacy in the target population: children between the ages of 9 months and 2 years. Knowing the seasonal pattern of rotavirus isolations in this country it would be possible to time the trials to coincide with the beginning of the epidemic season (weeks 37-40) to provide optimum challenge from wild virus.

In the last epidemic year in England and Wales at least 5800 infants and children under the age of 5 were affected by the diarrhoea, dehydration and discomfort of rotaviruses. Although precise data on numbers of hospitalisations are not available, extrapolating from prospective series in other countries suggests that the burden upon the health service would have been significant. Rotavirus vaccine presents an exciting prospect for reducing this morbidity. What is known about the epidemiology of rotaviruses, coupled with the results of the current vaccine trials, can be used to (1) predict the effect introduction of vaccine might have on the pattern of disease in our population, (2) construct a balance sheet for evaluating the vaccine, and if appropriate (3) guide the planning of a sensible vaccine programme.

I am grateful to Professor G Rose and Professor CS Peckham for their helpful advice and criticism during the preparation of this paper.

\section{References}

${ }^{1}$ World Health Organisation. Recent advances in knowledge of rotavirus diarrhoea. Report of the WHO scientific working group on viral diarrhoea. Geneva: 1982
${ }^{2}$ Vesikari T, Maki $M$, Isolauri E. Epidemiologic background for the need of rotavirus vaccine in Finland. Preliminary experience of RIT 4237 strain of live attenuated rotavirus vaccine in adults. Development Biological Standards 1983; 53: 229-236.

${ }^{3}$ DuPont $H$. Rotaviral gastroenteritis-some recent developments. J Infect Dis; 149: 663-666.

${ }^{4}$ Wolf JL, Schreiber DS. Rotavirus. Medical Clinics of North America 1982; 66: 580-584.

${ }^{5}$ Steinhoff, MC. Rotavirus: the first five years. $J$ Pediatr 1980; 96: 611-622.

6 Kapikain AZ, Wyatt RG, Greenberg HB, Kalica AR, Kim HW, Brandt CD, Rodriguez WJ, et al. Approaches to immunisation of infants and young children against gastroenteritis due to rotaviruses. Rev Infect Dis 1980; 2: 459-469.

${ }^{7}$ Hjelt K, Krasilnikoff A, Grauballe PC. Incidence of hospitalisation and outpatient clinical visits caused by rotavirus and non-rotavirus acute gastroenteritis. Dan Med Bull 1984; 31: 249-251.

${ }^{8}$ Monto AS, Koopman JS, Longini IM, Isaacson RE. The Tecumseh study. XII. Enteric agents in the community: 1976-1981. J Infect Dis 1983; 148: 284-291.

9 Pickering LK, Evans DG, DuPont HL, Vollet JJ, Evans DJ. Diarrhoea caused by shigella, rotavirus and giardia in day-care centres + prospective study. J Pediatr 1981; 99: 51-56.

10 Bartlett AV, Moore M, Gary GW, Stanko KM, Erben JJ, Meredith BA. Diarrhoeal illness among infants and toddlers in day care centres. J Pediatr 1985; 107 (4): 495-502.

${ }^{11}$ Grimwood K, Abbott GD, Fergusson DM, Jennings LC, Allan JM. Spread of rotavirus within families: a community based study. Br Med J 1983; 287: 575-577.

12 Sanders RC, Beards GM, Flewett TH. A shift in prevalent human rotavirus strains. Communicable Disease Report 1983; 83 (14): 3.

${ }^{13}$ Follett E, Saunders RC, Beards GM, Hundley F, Desselberger U. Molecular epidemiology of human rotaviruses. J Hygiene 1984; 92: 209-222.

${ }^{14}$ Lambert J, Marissens D, Marbehart P, Zissis G. Prevalence of subgroup 1, 2 and 3 rotaviruses in Belgian children suffering from acute diarrhoea.J Med Virol 1983; 11: $31-38$.

${ }^{15}$ Konno T, Sato T, Suzuki H, Kitaoka S, Katsushima N, Sakamoto M, Yazaki N, et al. Changing RNA patterns in rotaviruses of human origin: demonstration of a single dominant pattern at the start of an epidemic and various patterns thereafter. J Infect Dis 1984; 149: 683-687.

${ }^{16}$ Follett E, Desselberger U.. Cocirculation of different rotavirus strains in a local outbreak of infantile gastroenteritis. J Med Virol 1983; 11: 39-52.

${ }^{17}$ Champsaur H, Henry-Amar M, Goldszmidt D, Prevot J, Bourjouane M, Bach C. Rotavirus carriage, asymptomatic infection and disease in the first two years of life. II Serological response. J. Infect Dis 1984; 149 (5): 657-682.

${ }^{18}$ Champsaur H, Questiaux E, Prevot J, Henry-Amar M, Goldszmidt D, Bourjouane $M$, Bach C. Rotavirus carriage, asymptomatic infection, and disease in the first two years of life. I. Virus shedding. J Infect Dis 1984; 149 (5): 667-674.

${ }^{19}$ Weinberg RJ, Tipton G, Klish WJ, Brown MR. Effect of breast feeding on morbidity in rotavirus gastroenteritis. Pediatr 1984; 74: 250-253.

${ }^{20}$ Identification of viruses, chlamydias, rickettsias and mycoplasmas. OPCS Monitor MB2. 1982-85. 
${ }^{21}$ Vesikari T, Isolauri E, Delem A. Immunogenicity and safety of life oral attenuated bovine rotavirus vaccine strain RIT 4237 in adults and young children. Lancet 1983; ii: 807-811.

${ }^{22}$ Vesikari T, Isolauri E, d'Hondt E. Protection of infants against rotavirus diarrhoea by RIT 4237 attenuated bovine rotavirus strain vaccine. Lancet 1984; i: 977-800.

${ }^{23}$ Vesikari T, Isolauri E, Delem A. Increased "take" rate of oral rotavirus vaccine in infants after milk feeding. Lancet 1984; ii: 700.

${ }^{24}$ Vesikari T, Isolauri E, Delem A, d'Hont E, Andre FE, Beards GM, Flewett TH. Clinical efficacy of the RIT 4237 live attenated bovine rotavirus vaccine in infants vaccinated before a rotavirus epidemic. J Pediatr 1985; 107: 189-194. 